\title{
Condições periodontais e fatores associados a profundidade de sondagem em pacientes de uma faculdade do sul do Brasil
}

\section{Periodontal conditions and factors associated with the probing depth in patients from a faculty in southern Brazil}

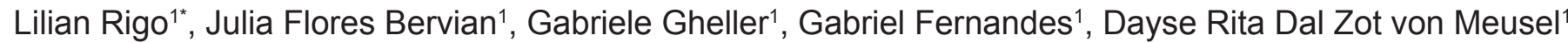

1. Faculdade Meridional - IMED, Passo Fundo, RS, Brasil

* Correspondencia Autor: Lilian Rigo | E-mail: Iillian. rigo@imed.edu.br | Telefone: +54999270441|

ORCID: 0000-0003-3725-3047

Trabajo recibido el 16/05/2020.

Aprobado para su publicación el 09/08/2020

\begin{abstract}
RESUMO
Objetivos: Verificar os sinais clínicos periodontais e investigar a os níveis de profundidade de sondagem (PS) e fatores associados em pacientes atendidos nas Clínicas da Faculdade IMED, localizada em um município do sul do Brasil. Metodologia: Os dados foram coletados em 193 prontuários de pacientes que buscaram atendimento odontológico, no período de 2017-2018, agrupados em sociodemográficos, hábitos de higiene bucal, hábitos nocivos e doenças crônicas. Os dados clínicos foram índice de placa dental visível (IPV), índice de sangramento gengival (ISG) e PS. Após realizadas análises descritivas foi construído um modelo de Regressão linear simples e múltiplo para verificar relações entre as variáveis ao $p$-valor $<0,05$. Resultados: A maioria dos pacientes são do sexo feminino $(63,2 \%)$ com média de 40,8 anos $( \pm 14,58)$. Os resultados descritivos de IPV, ISG e PS foram $39,11 \pm 32,28 \%, 21,81 \pm 22,43 \%$ e 2,91 $\pm 1,09 \mathrm{~mm}$, respectivamente. Após os ajustes no modelo de regressão múltiplo, os portadores de diabetes e a a quantidade de cigarros fumados por dia influenciaram no nível de PS. Conclusões: Os achados desta investigação mostram um alto IPV e de SG. Apesar da média da PS ter sido baixa, portadores de diabetes e fumo foram fatores preditivos de maiores níveis de PS.

DESCRITORES

Doença Periodontal; Gengivite; Índice Periodontal; Diabetes Mellitus; Doença cardiovasculares; Fumantes; Alcoólicos.
\end{abstract}

Int. J. Inter. Dent Vol. 13(3); 135-139, 2020.

\section{ABSTRACT}

Objectives: To evaluate periodontal clinical signs and investigate the probing depth levels (PD) and associated factors in patients attending the Dental Clinics of the Faculty IMED, located in a municipality in southern Brazil. Material and Methods: The data were collected of 193 records of patients who demand dental care in the Dental Clinics for periodontal treatment, in the period 2017-2018: sociodemographic data, oral hygiene habits, tobacco use and chronic diseases. The clinical data were: gingival bleeding index (GBI), visible plaque index (VPI) and PD. The statistical analyzed the relationship between probing depth and exposure variables, using a linear regression linear model ( $p$-value $<0.05)$. Results: The majority of the patients attended were female $(63.2 \%)$ and 40.8 years old on average $( \pm 14.58)$. The descriptive results of VPI, GBI e PD were: $39.11 \pm 32.28 \%, 21.81 \pm 22.43 \%$, and $2.91 \pm 1.09 \mathrm{~mm}$, respectively. After adjustments in the multiple regression model, it was observed that diabetes and quantity of cigarettes smoked had an influence on PD. Conclusions: The results obtained in this research allowed to verify a high index of VPI and GBI in the patients. The mean PD of the individuals was low, however, it was possible to verify that having diabetes and smoking a large amount of cigarettes daily were predictive factors of higher levels of PD.

\section{KEY WORDS}

Periodontal Disease; Gingivitis; Periodontal Index; Diabetes Mellitus; Cardiovascular Diseases; Smokers; Alcoholics.

Int. J. Inter. Dent Vol. 13(3); 135-139, 2020. 


\section{INTRODUÇÃO}

A doença periodontal (DP) é uma manifestação crônica que lesa os tecidos de proteção gengival (gengiva livre e gengiva aderida) e de sustentação dos dentes (cemento, ligamento periodontal e osso alveolar) $)^{(1-2)}$. É uma doença de grande importância para o indivíduo e um problema bucal de saúde pública, pois, como é uma resposta inflamatória destrutiva, quando não tratada, pode ocasionar a perda dental e diversos problemas ao organismo humano(2). A DP tem etiologia multifatorial, porém, o principal fator etiológico é a presença do biofilme microbiano aderido próximo aos tecidos periodontais a mais de 20 dias no sulco gengival(3).

O desenvolvimento das doenças periodontais pode ser influenciado por diversas condições, como: características próprias do indivíduo, fatores sociais e comportamentais, doenças, genética, anatomia dental, composição microbiológica do biofilme dental e outros fatores de riscos ${ }^{(4)}$. Em um estágio mais avançado das doenças periodontais ocorre a perda de inserção de maneira irreversível, podendo posteriormente levar a perda dental. Essa sequela da doença repercute negativamente na qualidade de vida do indivíduo, em razão do comprometimento das funções estomatognáticas, como a mastigação, a deglutição, a fonação e a estética(1).

Contudo, algumas doenças sistêmicas crônicas e hábitos viciosos podem estar diretamente às doenças do periodonto.

As doenças cardiovasculares e doença isquêmica cardíaca, de origem arterosclerótica, são um dos maiores problemas na saúde pública, tanto no Brasil como em outros países e o seu desenvolvimento tem sido associado a infecções periodontais, pois as bactérias anaeróbias situadas nas bolsas periodontais, se encontram em um habitat adequado para colonizar o endotélio via corrente sanguínea e incitar a agregação plaquetária, aumentando assim, as chances de um episódio trombogênico. A DP representa um aumento significativo para o risco de doenças cardiovasculares, devido às respostas inflamatórias da DP aos altos níveis de mediadores que podem exacerbar o processo de aterogênese ${ }^{(5)}$.

Outra enfermidade que pode estar associada a DP é a diabetes mellitus, que compreende uma deficiência metabólica reconhecida como hiperglicemia, causando falha na secreção ou ação da insulina. É dividida em dois tipos: tipo 1 (diabéticos insulino-dependentes), relacionada a destruição autoimune das células responsáveis pela produção de insulina e é diagnosticada em crianças e adolescente; tipo 2 está ligada a alteração na produção e resistência celular a insulina(6). A presença de DP pode causar o aumento sistêmico dos níveis de citocinas inflamatórias, que induzem a resistência a insulina, prejudicando o controle glicêmico da diabetes. O controle glicêmico está associado a presença e severidade da DP, bem como o grau de controle glicêmico da diabetes pode influenciar no tratamento da $\mathrm{DP}^{(6)}$.

A depressão é definida como um transtorno psiquiátrico incapacitante, manifestando-se com humor deprimido, sintomas vegetativos, deficiência cognitiva e potencial fator prejudicial a qualidade de vida e função física(7). Essa condição, que, atualmente, tem alta prevalência na população mundial, tornou-se alvo de pesquisas. Vários estudos vêm demonstrando a relação da DP com estresse e transtornos depressivos, destacando elevado nível de cortisol, negligente higiene oral, maiores níveis de PS, perda de inserção e perda de dentes ${ }^{(8)}$.

A DP se manifesta devido a uma interação do biofilme bacteriano (agressão microbiana) e com os mecanismos de defesa do hospedeiro(1). Porém, o tabagismo agrava os estágios de DP, causado pelo aumento da colonização de patógenos periodontais no biofilme subgengival de fumante ${ }^{(4)}$. Os indivíduos tabagistas apresentam uma menor redução de bactérias periodonto patogênicas em locais que receberam raspagem e alisamento radicular, podendo assim, justificar a pior resposta dos fumantes a terapia periodontal mecânica ${ }^{(4)}$. Dessa forma, hábitos nocivos como uso de tabaco e bebidas alcoólicas também podem estar associados a problemas relacionados ao periodonto.

A presente pesquisa torna-se importante pelo fato de não existirem muitos estudos que mostrem o perfil demográfico, os hábitos e a presença de problemas sistêmicos em pacientes que procuram atendimento em Clínicas Odontológicas. Assim, os objetivos do estudo foram verificar os sinais clínicos periodontais e investigar a os níveis de PS e fatores associados em pacientes atendidos nas Clínicas de uma Faculdade, a partir da análise de prontuários.

\section{METODOLOGIA}

A pesquisa foi submetida à aprovação do Comitê de Ética em Pesquisa (CEP) da Faculdade IMED e aprovado sob número 2.607.711, CAAE 87435018.4.0000.5319, na data de 18 de abril de 2018, tendo sido autorizada anteriormente pelo Coordenador das Clínica de Odontologia da Faculdade. O presente artigo científico foi redigido de acordo com as recomendações do reporte de STROBE (Strengthening the Reporting of Observational Studies in Epidemiology).

\section{Delineamento do estudo e amostra}

A pesquisa caracteriza-se como um estudo observacional de abordagem quantitativa, cujo delineamento é transversal.

A amostra do tipo probabilística foi composta por 193 prontuários devidamente preenchidos de pacientes a partir de 18 anos de idade que realizaram o atendimento odontológico nas Clínicas de Odontologia da Faculdade de Odontologia da IMED, Passo Fundo, Rio Grande do Sul (RS), Brasil, para tratamento periodontal.

Os registros da Faculdade possuem um total de 300 prontuários dos pacientes que realizaram tratamento periodontal no ano de 2017 e 240 no ano de 2018. Dessa forma, a fim de garantir uma amostra representativa foi realizado um cálculo amostral para uma amostragem probabilística sistemática dos prontuários dos dois anos.

Para o cálculo amostral com uma população total de 540 prontuários, utilizando nível de confiança de $90 \%$, margem de erro de $5 \%$, probabilidade de agravos de $50 \%$, o tamanho da amostra ficou em 225 prontuários. Os prontuários foram numerados iniciando pelo primeiro prontuário atendido do mês de marco de 2017 até o último prontuário de 2018, e alocados de três em três (Ex: escolhido o número 1, descartando 2 e 3, escolhendo o número 4, e assim, por diante).

Porém, em função de alguns prontuários terem sido excluídos por não estarem totalmente preenchidos, a amostra deste estudo finalizou em 193 prontuários, havendo uma perda amostral de 32 prontuários $(14,22 \%)$ e um total de $85,78 \%$ da amostra calculada.

\section{Localização do estudo}

O município de Passo Fundo está localizado no Norte do Rio Grande do Sul, sendo a maior cidade desta região. Possui uma população de 197.798 habitantes $^{(9)}$. O município é um dos polos de saúde do estado, possui nove hospitais, quatro Faculdades com cursos da área da saúde, sendo que três delas possuem curso de Odontologia. A Faculdade IMED é uma Instituição Privada em Ensino Superior, localizada no município de Passo Fundo, tendo sido fundada no ano de 2004 e deu início ao curso de Odontologia no ano de 2010. Este curso possui três Clínicas Odontológicas que prestam atendimento dentário à população de Passo Fundo e região.

\section{Procedimentos e instrumentos para coleta de dados}

A coleta de dados foi a partir de prontuários clínicos dos pacientes já atendidos ou ainda em atendimento na Faculdade no período de março de 2017 a novembro de 2018. Os dados foram coletados pelo pesquisador em dois meses (março e abril de 2019). Ainda como critérios de inclusão, os indivíduos tinham 18 anos ou mais.

Os prontuários da Faculdade de Odontologia foram preenchidos pelos alunos matriculados nas disciplinas de Clínicas Odontológicas do quarto ao sexto semestre do curso de Odontologia. Vale destacar que os alunos que realizam o atendimento estão devidamente treinados pelos professores das disciplinas em semestre teórico anteriormente cursado, além da supervisão dos professores da área com especialização em Periodontia. Os materiais utilizados para o exame clínico bucal são o espelho bucal e a sonda milimetrada de Williams (Millenium-Golgran ${ }^{\circledR}$ ) devidamente esterilizados, seguindo normas de biossegurança, sendo examinados em equipamentos odontológicos com luz artificial (refletor odontológico).

Primeiramente, foi avaliada a ficha de anamnese onde constam os dados sociodemográficos como: sexo, idade, estado civil, escolaridade, doenças crônicas auto reportadas pelo paciente (diabetes, doença cardiovascular e transtorno depressivo), fumo e alcoolismo. Dados sobre frequência de higiene bucal, tipo de escova dental uso de antisséptico também foram retirados da ficha anamnese.

Os dados clínicos bucais foram retirados das fichas específicas de Periodontia, que fazem parte do prontuário completo de exames dos pacientes. As mensurações clínicas analisadas no estudo foram: IPV, ISG e PS. O IPV considera a presença de placa visível em 4 faces dos dentes avaliando o controle de placa do paciente. O ISG verifica a ocorrência de sangramento na gengiva marginal após a sonda percorrer suavemente a extensão do sulco gengival em profundidade de 0,5 a 1 $\mathrm{mm}$. A PS, é identificada como a distância compreendida entre a margem gengival e a porção mais apical sondável da bolsa ou sulco, mensurada em milímetros.

\section{Variáveis em estudo}

Variáveis clínicas - relacionadas aos sinais clínicos de problemas 
periodontais: ISG, IPV e PS. Para esta pesquisa usou-se a variável dependente: PS - variável contínua.

Variáveis de exposição independentes: 1. Questões sociodemográficas: nível de escolaridade (Ensino Fundamental, Ensino Médio e Ensino Superior), sexo (masculino e feminino), idade e estado civil (solteiro, casado/união estável, separado/viúvo); 2. Questões de hábitos de higiene (frequência de escovação diária, tipo de escova dental - ultra macia/macia/ média/dura-, uso de antisséptico bucal - $\mathrm{sim} /$ não-); 3. Hábitos nocivos (fumante - sim/não-, quantidade de cigarros, alcoolistas (sim - ingere álcool 4 ou mais vezes por semana /não - nunca ingere ou até 3 vezes na semana-); 4. Doenças crônicas (diabetes, doenças cardiovasculares, transtornos depressivos - sim/não).

\section{Análise de dados}

Os dados obtidos foram organizados em planilhas de Excel e exportados para o programa estatístico IBM SPSS ${ }^{\circledR}$ software (Statistical Package for the Social Sciences), versão 20.0, Armonk, New York.

$\mathrm{Na}$ estatística descritiva foram verificadas as frequências absolutas e relativas, medianas, médias, desvios padrão e percentis das variáveis clínicas periodontais.

Para as medidas de IPV e do ISG foram somadas todas as faces dos dentes com presença de placa ou sangramento (4 faces) multiplicadas por 100 e divididas pelo número total de faces dos dentes presentes em cada indivíduo. Para avaliação dos valores de PS, aferidas em milímetros, foram somados todos os valores e divididos pelo número de dentes presentes em boca de cada um dos indivíduos. Depois disso, foram mensuradas a mediana, média, desvio padrão e percentis de cada uma das variáveis clínicas.

Foram testadas análises univariada entre as variáveis hábitos nocivos (quantidade de cigarros por dia e alcoolistas) e as doenças crônicas investigadas nos prontuários dos pacientes (diabetes, doenças cardiovasculares e transtorno depressivo) e o desfecho PS, porém, as doenças cardiovasculares não apresentaram significância estatística $(p>0,10)$, optando-se por não inserir no modelo final ajustado. Foram inseridas no modelo múltiplo, as demais variáveis: quantidade de cigarros por dia; alcoolista; diabetes; e transtorno depressivo. O modelo foi construído para verificar influência das variáveis de exposição destacadas anteriormente e a variável desfecho - PS - condição mais grave indicativa de problemas periodontais.

A análise estatística para a verificar relação entre PS e variáveis de exposição foi realizada por meio de Regressão linear simples e múltipla ao p-valor $<0,05$ e intervalos de confiança de $95 \%$, resultando em um modelo estatisticamente significativo.

\section{RESULTADOS}

Do total de 193 prontuários, $63,2 \%$ dos indivíduos eram do sexo feminino com idade entre 18 e 81 anos, média de 40,8 e desvio padrão (dp) de 14,6 . Em relação ao estado civil, a maioria era casado ou mantinha união estável e $61,7 \%$ já cursou o Ensino Médio. Os dados sociodemográficos estão descritos na Tabela 1.

A Tabela 2 descreve os dados das doenças crônica, os e hábitos nocivos e os de higiene bucal de todos os indivíduos da amostra. Em relação as doenças crônicas, 8,8\% relataram ter diabetes, $19,7 \%$ são portadoras de doenças cardiovasculares e $8,3 \%$ sofrem de transtorno depressivo. Quanto aos hábitos, $19,7 \%$ são fumantes, e destes $17 \%$ fumam de 5 a 20 cigarros por dia, e 2,5\% fumam de 25 a 50 de cigarros por dia. Quanto ao consumo de álcool, 26,4\% relataram ser alcoolistas. Foram computados os dados de hábitos de higiene presente nos prontuários, a fim de descrição. Em relação a escovação dentária, 72,5\% dos indivíduos relataram escovar os dentes 3 vezes por dia, sendo que $45,1 \%$ utilizam escova dental média e $39,9 \%$ as escovas dentais macias. Quanto ao bochecho, $38,9 \%$ relataram fazer bochecho com antisséptico rotineiramente.

Quanto as medidas descritivas dos sinais periodontais IPV, ISG e PS, os resultados mostram que a mediana do IPV foi de $29,46 \%$ e média de $30,11 \%$ (dp $32,28 \%$ ). A mediana do ISG foi de $15,38 \%$ e a média $21,81 \%$ (dp 22,43\%). A média da PS foi de 2,91 mm ( $d p 1,09 \mathrm{~mm})$. Os resultados encontram-se na Tabela 3.

O modelo de regressão testou a variável PS, hábitos nocivos e doenças crônicas. Após ajustes no modelo de regressão múltiplo observou-se que diabetes $(\beta=0,15$; IC95\%0,06-1,11) e quantidade de cigarros fumados $(\beta=-0,17$; IC95\% $(0,001-0,04)$ tiveram influência na PS, explicando essa influência em $15 \%$ e $17 \%$, respectivamente. As variáveis estiveram associadas no modelo bruto $(p<0,10)$, mas após o ajuste perderam a associação: transtornos depressivos e alcoolistas e as variáveis que se mantiveram associadas com $p<0,05$ foram somente quantidade de cigarros e ter diabetes (Tabela 4).

Tabela 1: Distribuição da frequência das variáveis sociodemográficas dos pacientes das Clínicas Odontológicas da Faculdade IMED, Passo Fundo, RS, Brasil, 2017 e 2018 ( $n=193)$

\begin{tabular}{l|c|c}
\hline \multicolumn{1}{c}{ Variáveis } & N & $\%$ \\
\hline Sexo & 71 & 36,8 \\
\hline Masculino & 122 & 63,2 \\
\hline Feminino & 58 & 30,1 \\
\hline Idade & 84 & 43,5 \\
\hline 18 a 30 anos & 51 & 26,4 \\
\hline 31 a 50 anos & 73 & 37,8 \\
\hline 51 anos ou mais & 99 & 51,3 \\
\hline Estado Civil & 21 & 10,9 \\
\hline Solteiro & 34 & 17,6 \\
\hline Casado/união estável & 119 & 61,7 \\
\hline Viúvo/separado & 40 & 20,7 \\
\hline Escolaridade & \multicolumn{2}{|l}{} \\
\hline Ensino fundamental & \multicolumn{2}{|l}{} \\
\hline Ensino médio &
\end{tabular}

Tabela 2: Distribuição das frequências das doenças crônicas e hábitos nocivos e de higiene bucal dos pacientes das Clínicas Odontológicas da Faculdade IMED, Passo Fundo, RS, Brasil, 2017 e 2018 ( $n=193$ )

\begin{tabular}{|c|c|c|}
\hline Variáveis & $\mathbf{N}$ & $\%$ \\
\hline \multicolumn{3}{|l|}{ Diabetes } \\
\hline Sim & 17 & 8,8 \\
\hline Não & 176 & 91,2 \\
\hline \multicolumn{3}{|c|}{ Doenças Cardiovasculares } \\
\hline Sim & 38 & 19,7 \\
\hline Não & 155 & 80,3 \\
\hline \multicolumn{3}{|c|}{ Transtorno depressivo } \\
\hline Sim & 16 & 8,3 \\
\hline Não & 177 & 91,7 \\
\hline \multicolumn{3}{|l|}{ Fuma } \\
\hline Sim & 38 & 19,7 \\
\hline Não & 155 & 80,3 \\
\hline \multicolumn{3}{|c|}{ Quantos cigarros por dia } \\
\hline 0 & 155 & 80,3 \\
\hline 5 a 20 & 33 & 17,0 \\
\hline 21 a 50 & 5 & 2,5 \\
\hline \multicolumn{3}{|l|}{ Alcoolista } \\
\hline Sim & 51 & 26,4 \\
\hline Não & 142 & 73,6 \\
\hline
\end{tabular}

Quantas vezes escova os dentes ao dia

\begin{tabular}{l|c|c}
\hline 1 vez & 6 & 3,1 \\
\hline 2 vezes & 47 & 24,4 \\
\hline 3 vezes & 140 & 72,5
\end{tabular}

Tipo de escova

\begin{tabular}{l|c|c}
\hline Dura & 22 & 11,4 \\
\hline Média & 87 & 45,1 \\
\hline Macia & 77 & 39,9 \\
\hline Ultra Macia & 7 & 3,6 \\
\hline \multicolumn{2}{|l|}{ Faz bochecho com antisséptico bucal } \\
\hline Sim & 75 & 38,9 \\
\hline Não & 118 & 61,1 \\
\hline
\end{tabular}


Tabela 3: Medidas descritivas (mediana, média, desvio padrão e percentis) dos sinais clínicos periodontais IPV, ISG e PS dos pacientes atendidos na Clínica Odontológica da IMED, Passo Fundo, RS, Brasil, 2017 e 2018 $(n=193)$.

\begin{tabular}{|c|c|c|c|c|}
\hline & Mediana & Média & $\begin{array}{l}\text { Desvio } \\
\text { Padrão }\end{array}$ & Percentis \\
\hline IPV & $29,46 \%$ & $39,11 \%$ & $32,28 \%$ & $\begin{array}{l}12,95 \%(25) \\
29,46 \%(50) \\
62,93 \%(75)\end{array}$ \\
\hline ISG & $15,38 \%$ & $21,81 \%$ & $22,43 \%$ & $\begin{array}{c}4,46 \%(25) \\
15,38 \%(50) \\
31,12 \%(75)\end{array}$ \\
\hline PS & - & $2,91 \mathrm{~mm}$ & $1,09 \mathrm{~mm}$ & $\begin{array}{l}2,20 \mathrm{~mm}(25) \\
2,75 \mathrm{~mm}(50) \\
3,42 \mathrm{~mm}(75)\end{array}$ \\
\hline
\end{tabular}

Tabela 4: Modelo de regressão linear simples e múltiplo para a variável de condição periodontal PS, Passo Fundo, RS, Brasil, 2017 e 2018.

\begin{tabular}{|c|c|c|c|c|}
\hline & $\begin{array}{c}\text { Bruto } \\
\beta(\text { (IC95\%) }\end{array}$ & p-valor & $\begin{array}{l}\text { Ajustado } \\
\beta \text { (IC95\%) }\end{array}$ & p-valor \\
\hline \multicolumn{5}{|c|}{ Diabetes } \\
\hline Não & 1 & \multirow[b]{2}{*}{0,029} & 1 & \multirow[b]{2}{*}{$<0,028$} \\
\hline Sim & $\begin{array}{c}0,15(0,06 ; \\
1,14)\end{array}$ & & $\begin{array}{c}0,15(0,06 ; \\
1,11)\end{array}$ & \\
\hline
\end{tabular}

Transtornos depressivos

\begin{tabular}{|c|c|c|c|c|}
\hline Não & 1 & & 1 & \\
\hline Sim & $\begin{array}{c}-1,24(-1,05 \\
-0,06)\end{array}$ & 0,085 & $\begin{array}{c}-0,09(-0,90 ; \\
-0,18)\end{array}$ & 0,198 \\
\hline
\end{tabular}

Doenças cardiovasculares

\begin{tabular}{l|c|l|l|l}
\hline Não & & \multirow{2}{*}{0,143} & - & - \\
\cline { 1 - 2 } $\operatorname{Sim}$ & $\begin{array}{c}0,10 \\
(0,09 ; 0,67)\end{array}$ & & & \\
\hline
\end{tabular}

\section{Alcoolista}

\begin{tabular}{|c|c|c|c|c|}
\hline Não & 1 & & 1 & \\
\hline Sim & $\begin{array}{c}0,01(0,09 ; \\
0,79)\end{array}$ & 0,012 & $\begin{array}{c}0,13(0,01 ; \\
0,67)\end{array}$ & 0,061 \\
\hline
\end{tabular}

Quantos cigarros/dia

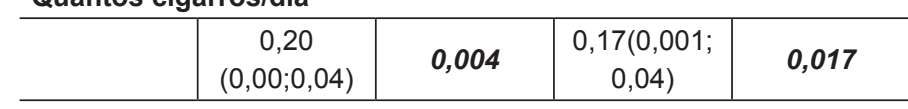

p-valor $<0,05$ - estatisticamente significativo

$\beta$ - coeficiente Beta; IC95\% - Intervalo de Confiança de 95\%

Ajustado pelas variáveis: diabetes, transtornos depressivos, doenças cardiovasculares, quantidade de cigarros/dia, alcoolista $(p<0,05)$.

\section{DISCUSSÃO}

Esta pesquisa verificou os sinais clínicos periodontais e a associação dos níveis de PS com doenças crônicas cardiovasculares, diabetes, transtornos depressivos, e hábitos nocivos, fumo e álcool, em pacientes atendidos nas Clínicas de uma Faculdade de Odontologia IMED, localizada no interior do Rio Grande do Sul, a partir da análise de prontuários no período de dois anos. $\mathrm{E}$ os achados mostraram que diabetes e fumo explicaram as alterações nas medidas de PS observadas nos indivíduos, confirmando alguns dados da literatura sobre a influência desses fatores para a problemas periodontais.

Quanto as medidas dos sinais clínicos IPV, ISG e PS foi possível verificar que os pacientes que frequentaram as Clínicas para atendimento odontológico tinham necessidade de tratamento, pois os índices de placa e de sangramento nas faces de todos os dentes apresentou-se elevados, com valores de $39,11 \pm 32,28 \%, 21,81 \pm 22,43 \%$, respectivamente, sendo que a média da PS foi baixa $(2,91 \pm 1,09 \mathrm{~mm})$. Em um estudo de coorte retrospectivo, o resultado do índice de placa foi mais alto $(68,78$ $\pm 20,56 \%$ ), porém, em relação a PS, os resultados se assemelharam ao do presente estudo $(2,97 \pm 0,71 \mathrm{~mm}) 10$. Observa-se que são achados interessantes, pois, no estudo em questão, os pesquisadores avaliaram a relação entre a DP e níveis lipídicos ao longo do tempo em pacientes que apresentavam doença arterial coronariana crônica ${ }^{(10)}$. Contudo, no presente estudo, a maioria dos pacientes avaliados eram saudáveis e adultos jovens.

Em um outro estudo que avaliou os pacientes atendidos em uma Clínica Odontológica de uma Faculdade, utilizando o Índice Periodontal Comunitário (IPC), os achados mostraram uma alta prevalência de cálculo dentário $(88,4 \%)$, seguido de sangramento gengival $(82,1 \%)^{(11)}$. Assim como, em um Levantamento Nacional com dados da população (SB Brasil 2010) realizados pelo Ministério da Saúde, observou-se alta prevalência de sangramento gengival e cálculo dentário em adolescentes e adultos jovens ${ }^{(12)}$.

No presente estudo houve associação estatisticamente significativa entre portadores de diabetes e maiores níveis de PS. Atualmente, se reconhece que a diabetes é uma doença prevalente na população mundial, principalmente em indivíduos com idade superior a 65 anos ${ }^{(13)}$. Há muito tempo, discute-se que diabetes corrobora com diversos problemas de saúde bucal, em especial, as alterações no periodonto, pois, pessoas com diabetes tendem a ter mais infecções periodontais do que aquelas sem a doença. Na literatura encontra-se dados de que a diabetes é um fator de risco para a DP, podendo aumentar sua gravidade, extensão e progressão da doença, observando que um pior controle glicêmico está diretamente relacionado a $\mathrm{DP}^{(13)}$. Paciente diabético controlado tem menor chance de desenvolver periodontite quando comparado a paciente diabético descompensado(13). Em outra pesquisa, $98,25 \%$ dos pacientes que apresentavam alterações sistêmicas (diabetes e/ou doenças cardiovasculares) foram diagnosticados com periodontite, constatando maior prevalência de periodontite agressiva ${ }^{(14)}$.

Quanto as doenças cardiovasculares, apesar de quase $20 \%$ serem portadores neste estudo, não houve associação significativa com níveis de PS no modelo ajustado. Entretanto, o seu desenvolvimento vem sendo frequentemente associado a infecções periodontais, pois as bactérias anaeróbias situadas nas bolsas periodontais, se encontram em um habitat adequado para colonizar o endotélio via corrente sanguínea e incitar a agregação plaquetária, aumentando, assim, as chances de um episódio trombogênico ${ }^{(5)}$. Guênes et al. ${ }^{(15)}$ analisaram a condição periodontal e a necessidade de tratamento em pacientes com doenças cardiovasculares no Centro Hospitalar João XXIII em Campina Grande (Paraíba), verificando que $53,1 \%$ dos sextantes apresentavam cálculos dentários e bolsa periodontal superior a 3,5 mm foi encontrada em $34,3 \%$ da amostra, concluindo mais alterações periodontais em pacientes com doenças cardiovasculares. Embora, não tenha mostrado uma associação entre PS e os pacientes com doenças cardiovasculares na presente pesquisa, observou-se uma importante prevalência de indivíduos portadores da doença crônica. Deve-se levar em consideração no momento das avaliações, a faixa etária dos indivíduos, pois, indivíduos com idades avançadas podem ter uma maior probabilidade de apresentar doenças cardiovasculares, sendo assim, o fator idade poderia explicar a associação com a presença de DP. Salienta-se ainda, que as doenças cardiovasculares e doença isquêmica cardíaca de origem arterosclerótica são um dos maiores problemas na saúde pública, tanto no Brasil como em outros países ${ }^{(5)}$.

Achados importantes nesta pesquisa evidenciaram uma associação significativa entre os indivíduos tabagistas que fumam maiores quantidades de cigarro por dia e maiores níveis de PS. O tabagismo vem sendo relacionado a DP e a principal consequência é a perda de elementos dentários subsequentes. Indivíduos que fumam cachimbos ou charutos sofrem efeitos no periodonto semelhantes aos que fumam cigarros $^{(13)}$. Khan et al..(16) observaram ma prevalência de $81,6 \%$ de periodontite em indivíduos fumantes no Paquistão, observando que os indivíduos tabagistas excessivos tiveram 3,5 vezes mais chances de desenvolver periodontite crônica do que os tabagistas moderados e leves. Khemis et al. ${ }^{(17)}$ avaliaram os efeitos do uso de narguilé no periodonto em comparação ao uso de cigarros em 148 indivíduos jovens e adultos, e verificaram que em comparação com os indivíduos que utilizavam somente narguilé, os tabagistas apresentavam significativamente maior profundidade de bolsa e índice gengival, ainda observando que todos os fumantes da amostra apresentavam mobilidade dentária, o que fez com que os autores concluíssem que tanto o uso de narguilé quanto o tabaco alteram a saúde bucal, porém, os tabagistas exclusivos e crônicos apresentaram mais efeitos prejudiciais no periodonto. No estudo de Mohanty et al. ${ }^{(18)}, 80$ tabagistas foram avaliados afim de comparar os níveis de biomarcadores em diferentes grupos de fumantes para o diagnóstico de periodontite e a sua relevância clínica, sendo que a hemoglobina livre foi maior em fumantes leves em comparação aos fumantes graves, concluindo que, a gravidade da DP aumentou conforme 
a gravidade do hábito de fumar aumentava. Muriandy et al. ${ }^{(19)}$ investigando a associação entre 330 tabagistas e DP na população que procurava tratamento periodontal, verificaram que $14,5 \%$ não estavam cientes de que fumar pode causar doenças gengivais. A proporção de fumantes que sabiam que o hábito poderia potencializar doenças gengivais foi consideravelmente menor em comparação aos não fumantes ${ }^{(19)}$. Franca et al. ${ }^{(20)}$ avaliaram a condição periodontal em indivíduos fumantes e não fumantes, observando maior recessão gengival e mobilidade dentária nos indivíduos fumantes, destacando a influência negativa do tabaco sobre a condição periodontal. Algumas das formas de DP se manifestam devido a uma agressão bacteriana ou interação com os mecanismos de defesa do hospedeiro, por sua vez, o tabagismo também é relacionado ao agravamento da DP, causado pelo aumento da colonização de patógenos periodontais no biofilme subgengival de fumante ${ }^{(13)}$.

No presente estudo não houve associação estatisticamente significativa com os indivíduos alcoolistas e maiores níveis de PS no ajuste do modelo final. Uma pesquisa de Lages et al.(21) observaram o efeito do consumo de álcool sobre os níveis de patógenos periodontais subgengivais e níveis de citocinas pró inflamatórias em indivíduos com e sem periodontite concluindo que os usuários dependentes de álcool apresentaram pior condição periodontal, bem como uma ligeira influência nos parâmetros imunológicos. Priyanka et al. ${ }^{(22)}$ avaliaram o impacto da dependência alcoólica na saúde bucal em alcoólatras em comparação a não alcoólatras, sendo que a prevalência de periodontite foi maior em dependentes alcoólicos em comparação aos controles. Manicone et al. ${ }^{(23)}$ avaliaram pacientes alcoolistas para identificar o efeito do consumo abusivo de álcool na saúde bucal concluindo que os pacientes alcoólatras e fumantes tiveram significativamente pior saúde bucal do que os não fumantes, incluindo problemas periodontais. Contudo, Sankaranarayanan et al. ${ }^{(24)}$, a fim de verificar a influência do álcool no desenvolvimento de bolsas periodontais durante um período de acompanhamento de 4 anos, não evidenciaram associação entre o consumo de álcool e o desenvolvimento de bolsas periodontais em população. Dessa forma, pode-se verificar, a partir das evidências relatadas, que o álcool pode estar associado ou não aos problemas periodontais. Ainda precisamos de mais evidências sobre o consumo de álcool, bem como a sua frequência, a fim de melhor compreender a sua associação com doenças periodontais.

No presente estudo, transtornos depressivos, foi uma doença crônica que não se manteve associada a PS no modelo final. Hsu et al. ${ }^{(7)}$ observaram que a incidência de depressão foi maior em pacientes com periodontite do que no grupo sem periodontite. Kumar et al. ${ }^{(8)}$ avaliaram a associação da depressão e periodontite em pacientes ambulatoriais de um Hospital e verificaram que a quantidade de placa e os níveis de PS foram significativamente maiores nos casos comparados aos controles.

Pode-se atribuir como uma limitação deste estudo, o fato do delineamento ser do tipo transversal, não podendo verificar causa e efeito por ser realizado em um único momento e, portanto, não identificando fatores de risco para os problemas periodontais aqui analisados. Outro fator limitador deve-se ao fato do próprio pesquisador não ter realizado o exame clínico bucal nos pacientes. Contudo, a pesquisa foi baseada em prontuários e todos eles foram devidamente preenchidos por discentes do curso de Odontologia com a supervisão de mestres e doutores na área da Periodontia.

Contudo, este estudo teve como objetivo avaliar os dados clínicos bucais dos pacientes de uma Faculdade, realizando para isso, uma coleta de dados metodologicamente adequada e dentro dos padrões rigorosos para pesquisas com desenhos transversais, apresentando resultados relevantes e associações que podem ser utilizadas para a tomada de decisão clínica de tratamento dos pacientes.

Destaca-se a importância de estudos longitudinais que possam fazer um acompanhamento a longo prazo destes pacientes atendidos nas Clínicas Odontológicas da Faculdade, podendo assim, identificar os fatores de risco para as doenças do periodonto e seus sinais clínicos. É notório a necessidade de mais pesquisas relacionadas ao consumo de álcool e a DP, bem como a relação de transtorno depressivo e DP, mesmo identificando nesta pesquisa que não houve uma associação.

\section{CONCLUSOES}

Os resultados obtidos nesta pesquisa permitiram verificar um alto IPV e ISG nos pacientes. A média de PS foi baixa, na maioria dos pacientes, o que não é considerada ainda uma DP.

Após ajuste no modelo de regressão linear múltiplo, foi possível verificar que, ter diabetes e o quantidade de cigarros fumados diariamente foram fatores preditivos de maiores medidas de PS

\section{CONFLITOS DE INTERESSES}

Os autores declaram não haver conflitos de interesse

\section{Referências}

1. Araújo GM, Sukekava F. Epidemiology of periodontal disease in Latin America. Periodontia. 2007;17(02):7-13

2. Lima HG, Lara VS. Immunological aspects of inflammatory periodontal disease: involvement of mast cells. UNOPAR Cient Ciênc Biol Saúde. 2013;15(3): 225-9.

3. Almeida HFV, Barros ALM, Andrade NK, Batista LHC. Evaluation of the occurrence of periodontal and gingival diseases among patients attended at a dental schoo clinic of a university center in the Northeast of Brazil. Braz J Periodontal. 2019; 29(1): 7-15.

4. Sallum AW, Cesar Neto JB, Sallum EJ. Tabagismo e a doença periodontal. Periodontia. 2007;17(2):45-53

5. Saba-Chujifi E, Santos-Pereira SA, Dias LZ. Interrelationship between periodonta diseases and ischemic cardiovascular and cerebrovascular diseases. Periodontia. 2007;17(2):21-31.

6. Novaes AB, Macedo GO, Andrade PF. Relationship between Periodontal disease and Diabetes Mellitus. Periodontia. 2007;17(02):39-44

7. Hsu CC, Hsu YC, Chen HJ, Lin CC, Chang KH, Lee CY, et al. Association of periodontitis and subsequent depression a nationwide population-based study. Medicine. 2015;94(51):e2347

8. Kumar A, Kardkal A, Debnath S, Lakshminarayan J. Association of periodontal health indicators and major depressive disorder in hospital outpatients. J Indian Soc Periodontol. 2015;19(5):507-11

9. IBGE. Instituto Brasileiro de Geografia e Estatística. Conheça as Cidade do Brasil. Passo Fundo. [Acesso em: 20 jan 2018]. Disponível em:< https://cidades. ibge.gov.br/brasil/rs/passofundo/panorama >

10. Kampits C, Rösing CK, Montenegro MM, Ribeiro IW, Saffi MA, Polanczyk CA, et al. Impact of periodontal disease in the lipid profile of patients with chronic coronary artery disease: a 3-year, retrospective cohort. Int J Cardiovasc Sci. 2016; 29(4):2709.

11. Gaio E, Franco A, Herval Álex M, Rodrigues RPCB, Bernardino Ítalo M, Paranhos $\mathrm{LR}$, et al. Multivariate analysis of periodontal disease and associated risk factors in a population of south Brazil. Acta Scientiarum. Health Sciences 2019;41(1):e45019. 12. Knack KC, Sabadin CE, Boclin KL, Oltramari ES, Portilio MN, Rigo L. Periodontal conditions in adolescents and young Brazilians and associated factors: Cross -sectional study with data from the Brazilian oral health survey, 2010. J Indian Soc Periodontol 2019; 23:475-83.
13. Genco RJ, Borgnakke WS. Risk factors for periodontal disease. Periodonto 2000. 2013;62(1):59-94

14. Rosa Junior WD, Camargo AC, Buono EA, Borelli Neto L. Evaluation of chronic and aggressive periodontitis in patients with diabetes and cardiovascular impairments attending the clinic of periodontology. Rec Odonto Univers Cidade de São Paulo. 2009; 21(3): 212-8.

15. Guênes GM, Guênes GT, Ribeiro Al, Dantas DC, Bento PM, Lins RD, et al. Analysis of periodontal status and need for treatment in cardiac patients. Scientia Medica (Porto Alegre). 2011;21(2): 49-54.

16.Khan S, Khalid T, Awan KH. Chronic periodontitis and smoking. Prevalence and dose-response relationship. Saudi Med J. Aug 2016; 37(8): 889-94.

17. Khemiss M, Fekih DB, Khelifa MB, Saad HB. Comparison of periodontal status between male exclusive narghile smorkers and male exclusive cigarette smokers. Am J Mens Health. 2019;1-15.

18.Mohanty P, Gujjari SK, Nakum CG. Salivary free hemoglobin and lactate dehydrogenase as biomarkers for periodontal disease in smokers. Quintessence Int. 2019; 50(6): 428-34

19.Muniandy S. Knowledge on smoking and periodontal disease: A cross-sectional survey among targeted respondents. J Indian Soc Periodontol 2019;23(3):275-80.

20.Franca MS, Gomes RC, Lins RD, Dos Santos PA, Lima FJ. The influence of smoking on periodontal status. Stomatos 2010; 16 (31):23-36.

21. Lages EJ, Costa FO, Cortelli SC, Cortelli JR, Cota LO, Cyrino RM, et al. Alcoho Consumption and Periodontitis: Quantification of Periodontal Pathogens and Cytokines. J Periodontol. 2015;86(9):1058-68.

22. Priyanka K, Sudhir KM, Reddy VCS, Kumar RK, Srinivasulu G. Impact of alcohol dependency on oral health - a cross-sectional comparative study. J Clin Diagn Res. 2017;11(6):43-46.

23. Manicone PF, Tarli C, Mirijello A, Raffaelli L, Vassallo GA, Antonelli M, et al Dental health in patients affected by alcohol use disorders: a cross-sectional study. Eur Rev Med Pharmacol Sci. 2017: 21(22):5021-7.

24. Sankaranarayanan R, Saxlin T, Ylöstalo P, Khan S, Knuuttila M, Suominen AL. Alcohol use and periodontal pockets development: finding from a 4-yr longitudinal study. Eur J Oral Sci. 2019; 127(3):232-40. 